\title{
Assessment of bacterial diversity in breast milk using culture-dependent and culture-independent approaches
}

\author{
Ted Jost $^{1}$, Christophe Lacroix ${ }^{1 *}$, Christian Braegger ${ }^{2}$ and Christophe Chassard ${ }^{1}$ \\ ${ }^{1}$ Laboratory of Food Biotechnology, Institute of Food, Nutrition and Health, ETH Zurich, Schmelzbergstrasse 7 , \\ 8092 Zurich, Switzerland \\ ${ }^{2}$ Division of Gastroenterology and Nutrition, University Children's Hospital Zurich, Steinwiesenstrasse 75 , \\ 8032, Zurich, Switzerland \\ (Submitted 30 August 2012 - Final revision received 28 January 2013 - Accepted 28 January 2013 - First published online 14 March 2013)
}

\section{Abstract}

Initial neonatal gut colonisation is a crucial stage for developing a healthy physiology, beneficially influenced by breast-feeding. Breast milk has been shown not only to provide nutrients and bioactive/immunological compounds, but also commensal bacteria, including gut-associated anaerobic Bifidobacterium spp. The aim of the present study was to investigate bacterial diversity in breast milk, with emphasis on identifying gut-associated obligate anaerobes. Breast milk collected from seven mothers at three sampling points (days 3-6, 9-14 and 25-30 postpartum) was analysed by combined culture-dependent and state-of-the-art, culture-independent methods (Sanger sequencing and 454-pyrosequencing). In addition to the predominance of facultative anaerobes such as Staphylococcus, Streptococcus and Propionibacterium ( $>90 \%$ of isolated strains and $23.7 \%$ relative abundance using pyrosequencing), significant populations of obligate anaerobes, including Bifidobacterium and Veillonella, were detected using pyrosequencing and confirmed by the isolation of viable strains $(3.4 \%$ of isolates and $1.4 \%$ relative abundance). Pyrosequencing also revealed the presence of DNA of multiple major gut-associated obligate anaerobes (6.2\% relative abundance) such as Bacteroides and, for the first time, several members of the Clostridia, including butyrate producers, such as Faecalibacterium and Roseburia, which are important for colonic health. The present study suggests that breast milk may be a major source of bacterial diversity to the neonatal gut, including gut-associated obligate anaerobes, and may thus significantly influence gut colonisation and maturation of the immune system.

\section{Key words: Breast milk diversity: Neonatal gut microbiota: Gut-associated anaerobes: 454-Pyrosequencing}

Exclusive breast-feeding has long been associated with lower morbidity and mortality during the first year of life, as well as with reduced incidence and severity of infections and immune-related diseases, compared with formula feeding ${ }^{(1)}$. Breast milk not only provides essential nutrients and contains a broad range of bioactive, immunological and protective compounds unequalled by current formulae ${ }^{(1)}$, but has moreover been recognised as a continuous source of commensal and potential probiotic bacteria, able to influence initial neonatal gut colonisation and maturation of the immune system $^{(2)}$. Thus, understanding the impact of breast milk on early host-microbe interactions may provide future opportunities for modulating a dysbiotic microbiota and reduce the risk of disease ${ }^{(3)}$.

Predominant culturable bacterial populations in breast milk have been identified as facultative anaerobic members of the Bacilli (Firmicutes phylum), i.e. Staphylococcus and
Streptococcus spp. ${ }^{(4,5)}$. At the subdominant level, a range of facultative anaerobic strains have been isolated, including members of the Actinobacteria phylum (e.g. Propionibacterium and Rothia spp. $)^{(4,6)}$, members of the Bacilli, comprising lactic acid bacteria (e.g. Enterococcus and Lactobacillus spp.) ${ }^{(5,7)}$, and occasionally members of the Enterobacteriaceae (Proteobacteria phylum $)^{(6,8)}$. Because many culture-dependent studies were conducted in view of isolating potential probiotics, breast milk was furthermore shown to harbour obligate anaerobic gut-associated Bifidobacterium spp. ${ }^{(5,6,9-12)}$, which besides Bacteroides spp. represent the major anaerobic population of early gut microbiota in breast-fed neonates ${ }^{(13)}$. However, until now, little work has been performed on the detection of other viable major gut-associated anaerobes in breast milk, i.e. members of the Bacteroidetes phylum and the Clostridia class (Firmicutes phylum).

Abbreviations: cfu, colony-forming unit; LAMVAB, Lactobacillus anaerobic de Man, Rogosa and Sharpe agar with vancomycin and bromocresol green; MC, MacConkey agar no. 2; rRNA, ribosomal RNA; WC, Wilkins-Chalgren anaerobe agar.

* Corresponding author: C. Lacroix, fax +41 44 6321403, email christophe.lacroix@hest.ethz.ch 
Culture-independent methods have moreover begun to reveal the presence of DNA belonging to gut-associated anaerobic bacterial taxa not detected previously in breast milk in culture-dependent studies ${ }^{(8,14-17)}$. PCR-denaturing gradient gel electrophoresis, cloning and $16 \mathrm{~S}$ ribosomal RNA (rRNA) sequencing have led to the detection of the genera Bacteroides, Clostridium, Eubacterium and Veillonella in breast milk ${ }^{(8,15)}$. In addition, quantitative PCR analyses allowed for the quantification of the Bacteroides group and different groups of the Clostridia, including the Clostridium clusters IV and XIV that comprise the major butyrate producers important for colonic health (i.e. Faecalibacterium and Roseburia, respectively) ${ }^{(14,16)}$. Recently, high-throughput sequencing (i.e. 454-pyrosequencing) has been carried out on breast milk samples collected from 1 to 10 months postpartum, which allowed gaining the broadest insight into bacterial diversity in breast $\operatorname{milk}^{(16,17)}$. In both studies, facultative anaerobic members of the Bacilli accounted for the majority of DNA pyrosequences, but at a lower taxonomic level, large differences in relative abundance were apparent. While Staphylococcus and Streptococcus were predominant in the study by Hunt et al. ${ }^{(17)}$, which is supported by culture-dependent research ${ }^{(4,5)}$, in the study by Cabrera-Rubio et al. ${ }^{(16)}$, Weissella and Leuconostoc predominated over these genera. Despite these differences, which may be due to geographical and/or methodological factors, in both studies, sequences belonging to the obligate anaerobic genera Prevotella (Bacteroidetes phylum) and Veillonella (Clostridia class) were detected. However, although some obligate gut-associated anaerobes have been detected in breast milk using cultureindependent methods, no information about the viability of these populations could be gained. Thus, without confirming the presence of such members of the Bacteroidetes and Clostridia by culture and isolation of corresponding strains, it remains unclear whether breast milk is a source of such gutassociated anaerobes influencing initial gut colonisation, or whether dead cells or parts thereof are transferred to the breast-fed neonate. Therefore, the purpose of the present study was to investigate bacterial diversity in breast milk, emphasising on the identification of gut-associated anaerobes by combining aerobic and anaerobic culture methods with state-of-the-art, culture-independent methods, including Sanger sequencing and high-throughput sequencing (i.e. 454-pyrosequencing)

\section{Experimental methods}

\section{Subjects, study design and sample collection}

Healthy mothers-to-be, carrying a healthy baby and planning to deliver vaginally at term and to exclusively breast-feed during the postpartum period, were recruited for the present observational clinical study at the University Children's Hospital Zurich and the Hospital Zollikerberg (Zurich, Switzerland). Exclusion criteria were preterm and/or caesarean delivery, any formula feeding in addition to breast-feeding, as well any variables known to affect the balance of maternal (gut) microbiota, such as gastrointestinal and immunological disorders, and drug administration (e.g. antibiotics, laxatives) during the neonatal period and at least 4 months before delivery. The present study was conducted according to the guidelines laid down in the Declaration of Helsinki, and all procedures involving human subjects were approved by the Ethics Committee of the Canton of Zurich (Zurich, Switzerland; project identification code: StV 47/08; date of approval: 12 October 2009). Written informed consent was obtained from all subjects.

Breast milk samples were collected from seven mothers at three sampling points, i.e. at days 3-6, 9-14 and 25-30 postpartum. Due to the stringent inclusion criteria, two mothers were excluded from the study after the first sampling point and another twelve prepartum, mainly after caesarean delivery and/or antibiotic treatment. After rejection of foremilk and cleaning of the breast with aseptic soap, milk was collected using a hospital-grade electrical breast pump sterilised by autoclaving (Symphony). To minimise the exposure to $\mathrm{O}_{2}$, $5-15 \mathrm{ml}$ of breast milk were injected with a sterile syringe into a rubber-stoppered $100 \mathrm{ml}$ vessel devoid of air by flushing with $\mathrm{CO}_{2}$ before sterilisation. Samples were transported at $4^{\circ} \mathrm{C}$ and processed within $4 \mathrm{~h}$ in an anaerobic chamber (Coy Laboratory Products, Inc.) with an atmosphere of $85 \% \mathrm{~N}_{2}, 10 \%$ $\mathrm{CO}_{2}$ and $5 \% \mathrm{H}_{2}$ (PanGas AG). Aliquots of $2 \mathrm{ml}$ were immediately subjected to culture, while further aliquots were stored at $-80^{\circ} \mathrm{C}$ before DNA extraction and 454-pyrosequencing analysis.

\section{Culture and isolation}

For the quantification of viable bacterial populations in breast milk and the subsequent isolation of strains, aliquots of $100 \mu \mathrm{l}$ were plated in duplicate on two non-selective and seven selective agar media. The following media targeting anaerobic, major gut-associated bacterial populations were incubated in an anaerobic chamber: Bacteroides mineral salts agar for Bacteroides spp. (using $5 \mathrm{~g} / \mathrm{l}$ of D-glucose as the carbon source; VWR International) ${ }^{(18)}$, Beerens agar for Bifidobacterium spp. ${ }^{(19)}$, reinforced clostridial agar for members of the Clostridia $^{(20)}$ and Wilkins-Chalgren anaerobe agar (WC) for total anaerobes (Oxoid AG; supplemented with $0.5 \mathrm{~g} / 1$ of L-cysteine-HCl; Sigma-Aldrich). On the other hand, media targeting facultative anaerobic populations, i.e. MacConkey agar no. 2 (MC) for Enterobacteriaceae/Enterococcus spp. (Oxoid), mannitol salt agar for Staphylococcus spp. (Oxoid) and nutrient agar for total facultative anaerobes (Oxoid), were incubated aerobically. The Lactobacillus anaerobic de Man, Rogosa and Sharpe agar with vancomycin and bromocresol green (LAMVAB) targeting Lactobacillus spp. ${ }^{(21)}$ and azide blood agar for gram-positive cocci/Streptococcus spp. (Oxoid) were incubated in anaerobic jars. All plates were incubated at $37^{\circ} \mathrm{C}$ for $2-14 \mathrm{~d}$ and population levels are reported as log colony-forming units $(\mathrm{cfu}) / \mathrm{ml}$ breast milk.

Based on different morphologies, a set of colonies was isolated per sample and agar medium, streaked for purity and cultured in liquid media, i.e. Wilkins-Chalgren anaerobe broth for presumptive anaerobes (Oxoid; supplemented with $0.5 \mathrm{~g} / \mathrm{l}$ of L-cysteine- $\mathrm{HCl}$; Sigma-Aldrich), tryptone soya 
broth for facultative anaerobes (Oxoid) and de Man, Rogosa and Sharpe broth for presumptive Lactobacillus spp. (LaboLife Sàrl; supplemented with $0.5 \mathrm{~g} / 1$ of L-cysteine-HCl; Sigma-Aldrich). Purity was verified microscopically and viable isolates were maintained at $-80^{\circ} \mathrm{C}$ in $20 \%(\mathrm{v} / \mathrm{v})$ glycerol, while in addition, cell pellets obtained by centrifugation $(8000 \mathrm{~g}, 10 \mathrm{~min})$ were stored at $-20^{\circ} \mathrm{C}$ before DNA extraction and Sanger sequencing.

\section{DNA extraction}

DNA was extracted from pure culture cell pellets for subsequent Sanger sequencing using a Wizard Genomic DNA purification kit (Promega AG) according to the manufacturer's instructions. For pyrosequencing analysis, total DNA was extracted from pellets obtained by centrifugation ( $8000 \mathrm{~g}, 20 \mathrm{~min}$ ) of $1 \mathrm{ml}$ aliquots of breast milk, which were resuspended in sodium phosphate buffer (MP Biomedicals), using a FastDNA SPIN Kit for Soil and the FastPrep-24 instrument for mechanical lysis (MP Biomedicals) according to the manufacturer's instructions. DNA concentration and quality were assessed spectrophotometrically (NanoDrop 1000; Witec AG) and stored at $-20^{\circ} \mathrm{C}$ before molecular analyses.

\section{Sanger sequencing}

PCR amplification of near full-length 16S rRNA genes was performed using a 4:1 mixture of forward primers $8 f\left(5^{\prime}\right.$-AGAGTTTGATCMTGGCTCAG-3', universal) and 8f-bif (5'-AGGGTTCGATTCTGGCTCAG-3', Bifidobacterium-specific) and a universal bacterial reverse primer 1391R (5'-GACGGGCGGTGTGTRCA-3') (Microsynth AG) as described previously ${ }^{(22)}$. Reactions of $50 \mu \mathrm{l}$ contained $25 \mu \mathrm{l}$ of $2 \times$ MasterMix (Fermentas $\mathrm{GmbH}$ ), $0 \cdot 2 \mu \mathrm{M}$ of each primer (-mixture) and $1 \mu \mathrm{l}$ of template DNA diluted to $1 \mathrm{ng} / \mu \mathrm{l}$. Thermocycling (Biometra TProfessional Thermocycler; Biolabo Scientific Instruments SA) was performed with an initial denaturation step at $94^{\circ} \mathrm{C}$ for $300 \mathrm{~s}$, followed by thirty cycles of denaturation at $94^{\circ} \mathrm{C}$ for $30 \mathrm{~s}$, annealing at $57^{\circ} \mathrm{C}$ for $60 \mathrm{~s}$ and extension at $72^{\circ} \mathrm{C}$ for $30 \mathrm{~s}$ and a final extension at $72^{\circ} \mathrm{C}$ for $420 \mathrm{~s}$. Specificity and amplicon size were verified by electrophoresis in $1.5 \%(\mathrm{w} / \mathrm{v})$ agarose gels, and reactions were purified using an illustra GFX PCR DNA and Gel Band Purification Kit (GE Healthcare Europe $\mathrm{GmbH}$ ) according to the manufacturer's instructions.

Cycle sequencing PCR was carried out in $20 \mu \mathrm{l}$ reaction volumes with 5\% (v/v) BigDye v3.1 (Applied Biosystems Europe BV), $4 \mu \mathrm{l}$ of $5 \times$ sequencing buffer (Applied Biosystems), $1 \mu \mathrm{M}$ of reverse primer $1391 \mathrm{R}$ and $1 \mu \mathrm{l}$ of purified PCR template. Thermocycling (labcycler; SensoQuest $\mathrm{GmbH}$ ) was performed with an initial denaturation step at $96^{\circ} \mathrm{C}$ for $300 \mathrm{~s}$, followed by thirty-five cycles of denaturation at $96^{\circ} \mathrm{C}$ for $10 \mathrm{~s}$, annealing at $55^{\circ} \mathrm{C}$ for $20 \mathrm{~s}$ and extension at $60^{\circ} \mathrm{C}$ for $240 \mathrm{~s}$. Reactions were purified by dextran gel bead filtration (Sephadex; GE Healthcare) before loading $10 \mu \mathrm{l}$ for capillary electrophoresis (ABI 3130xl DNA Analyzer; Applied Biosystems). Sequencing trace chromatograms were quality-trimmed and checked for miscalled bases using a chromatogram viewer (FinchTV v1.4.0; Geospizia, Inc.). The Basic Local Alignment Search Tool algorithm $^{(23)}$ was used to align sequences with the GenBank database $^{(24)}$, and phylogenetic assignments were based on the nearest neighbour ( $\geq 97 \%$ sequence similarity), excluding sequences deposited from uncultured samples.

\section{Pyrosequencing}

High-throughput sequencing of total DNA extracted from breast milk was carried out at DNAVision SA (Charleroi, Belgium) using a 454 Life Sciences system in combination with Titanium chemistry (Roche AG).

Partial 16S rRNA genes were amplified by PCR using a forward primer containing the Titanium A adaptor sequence (5'-CCATCTCATCCCTGCGTGTCTCCGACTCAG-3'), a 5-10 nt multiplex identifier sequence and a templatespecific primer sequence. The reverse primer contained the Titanium B adaptor sequence (5'-CCTATCCCCTGTGTGCCTTGGCAGTCTCAG-3') and a template-specific primer sequence. Template-specific primer sequences (5'-AGGATTAGATACCCTGGTA- $3^{\prime}$ and 5'-CRRCACGAGCTGACGAC- $3^{\prime}$ ) allowed targeting the V5-V6 hypervariable $16 \mathrm{~S}$ rRNA region ${ }^{(25)}$. Each reaction mixture of $100 \mu \mathrm{l}$ contained $20 \mu \mathrm{l}$ of $5 \times$ KAPA HiFi Fidelity buffer, 2U of KAPA HiFi Hotstart DNA polymerase, $0.3 \mathrm{~mm}$ of each deoxyribonucleotide triphosphate (Kapa Biosystems), $0 \cdot 3 \mu \mathrm{M}$ of each primer (Eurogentec) and $60 \mathrm{ng}$ of template DNA. Thermocycling was performed with an initial denaturation step at $95^{\circ} \mathrm{C}$ for $5 \mathrm{~min}$, followed by twenty-five cycles of denaturation at $98^{\circ} \mathrm{C}$ for $20 \mathrm{~s}$, annealing at $56^{\circ} \mathrm{C}$ for $40 \mathrm{~s}$ and extension at $72^{\circ} \mathrm{C}$ for $20 \mathrm{~s}$, with a final extension of $5 \mathrm{~min}$ at $72^{\circ} \mathrm{C}$. Specificity and amplicon size were verified by electrophoresis in $1 \%(\mathrm{w} / \mathrm{v})$ agarose gels, and amplicons were purified using a Wizard SV Gel and PCR Clean-up System (Promega) according to the manufacturer's instructions.

Amplicons were quantified using a Quant-iT PicoGreen dsDNA assay kit (Life Technologies Corporation) according to the manufacturer's instructions and combined in equimolar concentrations for multiplexing. The final pool of DNA was purified using an Agencourt AMPure XP system (Agencourt Bioscience Corporation), according to the manufacturer's instructions, and resuspended in $100 \mu \mathrm{l}$ of Tris-EDTA buffer. Unidirectional pyrosequencing was then carried out using Primer A on a 454 Life Sciences Genome Sequencer GS FLX instrument (Roche) following Titanium chemistry.

Sequence quality was then verified according to the criteria: a maximum of one mismatch in the barcode and primer, a length of at least $240 \mathrm{nt}$ and a maximum of two undetermined bases per sequence (excluding barcode and primers). Phylum-, family- and genus-level taxonomic assignments of sequences that passed quality control were made using the Ribosomal Database Project Classifier (version 2.1; Center for Microbial Ecology, Michigan State University; http://rdp.cme.msu.edu/) ${ }^{(26)}$ with a confidence threshold of $80 \%$. The Mothur software package (Department of Microbiology and Immunology, University of Michigan; http://www.mothur.org/) was used for nearestneighbour clustering of the sequences into operational taxonomic units, based on which Chao1 richness and Shannon diversity estimations were calculated ${ }^{(27,28)}$. 


\section{Statistical analysis}

Quantitative data were obtained from culture (duplicates), averaged and $\log _{10}$-transformed. Mean log-transformed values and relative abundance data from pyrosequencing were calculated at each time point (means and standard deviations). Means were compared using Student's $t$ test and non-parametric Wilcoxon-Kruskal-Wallis tests with significance levels of $P<0.05$ using JMP statistical software (version 9; SAS Institute, Inc.).

\section{Results}

\section{Culture, isolation and strain typing}

Breast milk harboured low mean viable bacterial counts $<\log$ $3 \mathrm{cfu} / \mathrm{ml}$, as detected on the non-selective agar media, WC and nutrient agar, targeting total anaerobes $(\log 2.5$ (sD 0.9) cfu/ml; range $\log 1 \cdot 1-4.4 \mathrm{cfu} / \mathrm{ml}$ ) and total facultative anaerobes $(\log 2 \cdot 1(\mathrm{sD} 1 \cdot 1) \mathrm{cfu} / \mathrm{ml}$; range $\log 0-4 \cdot 2 \mathrm{cfu} / \mathrm{ml})$, respectively. Culture on seven selective media, however, revealed the predominance of presumptive Staphylococcus and Streptococcus populations, and low levels of presumptive Bifidobacterium, Enterobacteriaceae/Enterococcus and Clostridia populations, whereas no presumptive Bacteroides or Lactobacillus populations were detected on the respective selective agar media (Fig. 1). No significant trends of changes of bacterial population densities were detected within the first month postpartum (results not shown).

Following quantification of viable populations in each of the three breast milk samples obtained from the seven mothers, a total of 233 strains were isolated from the different agar media (sixty-two to eighty-six isolates per time point) based on different morphologies. Bacterial isolates were subjected to $16 \mathrm{~S}$ rRNA gene sequencing, which after quality

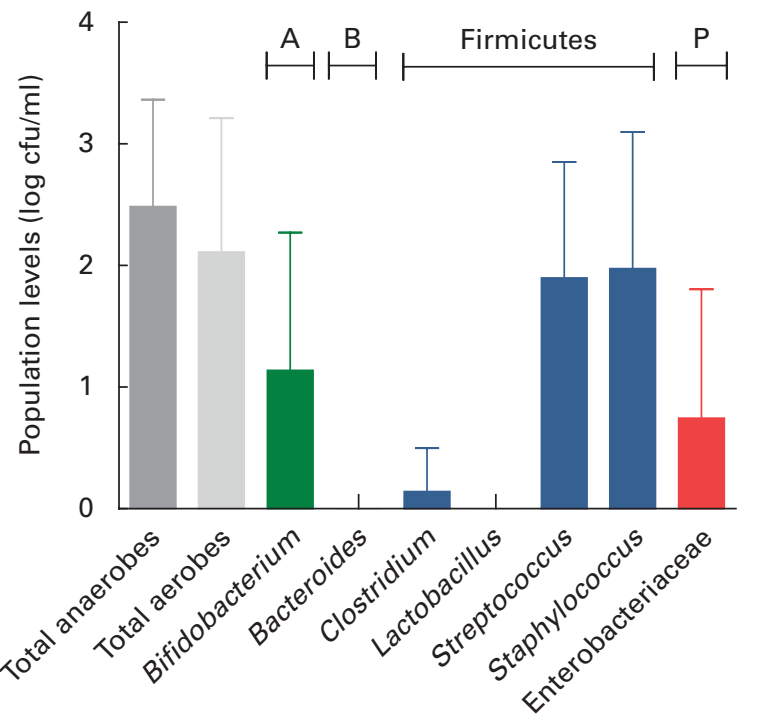

Fig. 1. Presumptive bacterial populations detected in breast milk collected from seven mothers at three sampling points, using culture on non-selective and selective agar media. Values are means, with standard deviations represented by vertical bars ( $n$ 21). A, Actinobacteria; B, Bacteroidetes; $\mathrm{P}$ Proteobacteria; cfu, colony-forming units. trimming resulted in partial sequences of 712 (SD 212) bp. Alignment of the sequences with those deposited in the GenBank database revealed that the majority of strains isolated from breast milk were members of the Firmicutes and Actinobacteria phyla, while members of Proteobacteria or Bacteroidetes were few or not detected, respectively. Over $90 \%$ of the strains isolated from breast milk belonged to three facultative anaerobic genera, Staphylococcus (60.1\%), Streptococcus (17.6\%) and Propionibacterium (13.7\%). More specifically, at the species level, Staphylococcus epidermidis, Streptococcus salivarius/thermophilus/vestibularis and Propionibacterium acnes strains were isolated from the breast milk samples of all seven mothers. An overview of the taxonomic assignment of the isolated strains, their isolation frequency in relation to the total amount of isolates, as well as subjectspecific prevalence are given in Table 1.

As revealed by the partial $16 \mathrm{~S}$ rRNA sequencing of isolates, the selective culture media did not always support the growth of the respective target populations. For instance, although presumptive Staphylococcus and Streptococcus populations were quantified at equally high levels (Fig. 1), isolates from azide blood agar comprised not only the strains of Streptococcus, but also the strains of Staphylococcus and Bifidobacterium. Likewise, the presumptive Enterobacteriaceae/Enterococcus population detected with MC agar was overestimated (Fig. 1), as beyond expectations, the growth of Staphylococcus spp. was not inhibited on this medium. No growth was detected on the highly selective LAMVAB agar targeting Lactobacillus spp.; however, few strains of Lactobacillus were isolated from both WC and reinforced clostridial agar agars. Similarly, strains isolated from Beerens agar targeting Bifidobacterium spp. were identified as Propionibacterium and Streptococcus spp., but strains of Bifidobacterium were isolated from the non-selective WC agar. Furthermore, few strains belonging to the genus Rothia, the opportunistic pathogen Klebsiella, as well as of the obligate anaerobic, lactate utiliser Veillonella, were isolated from the non-selective WC agar.

\section{Pyrosequencing}

High-throughput sequencing performed on breast milk DNA generated a total of 106075 quality-filtered, taxonomically classifiable 16S rRNA gene sequence reads with a mean of 5051 (SD 1523) reads per sample and a mean read length of 258 (SD 1) bp. The mean number of observed operational taxonomic units was 512 (SD 237) at 3\% similarity cut-off, based on which richness (Chao1 index: 1257 (SD 613)) and diversity (non-parametric Shannon index: 3.73 (SD 1.05)) were estimated.

Using the Ribosomal Database Project Classifier, sequences were assigned to the closest related taxa with a similarity threshold of $80 \%$. Mean relative abundances at the phylum level for each of the three breast milk sampling points revealed the predominance of four major phyla (Fig. 2). The Firmicutes and Proteobacteria phyla were the most abundant (approximately 42 and 35\%, respectively), followed by the Actinobacteria and Bacteroidetes phyla (approximately 9 and 
Table 1. Taxonomy of strains ( $n$ 233) isolated from breast milk of seven mothers, based on closest relatives ( $\geq 97 \%$ sequence similarity) using $16 \mathrm{~S}$ ribosomal RNA gene sequence alignments with GenBank

\begin{tabular}{|c|c|c|c|c|}
\hline \multirow[b]{2}{*}{ Phylum/genus } & \multirow[b]{2}{*}{ Species } & \multicolumn{2}{|c|}{ Isolation frequency* } & \multirow[b]{2}{*}{ Isolation medium } \\
\hline & & $\%$ & Prevalence & \\
\hline \multicolumn{5}{|l|}{ Actinobacteria } \\
\hline Bifidobacterium sp. $\dagger$ & B. breve† & 1.7 & $2 / 7$ & WC, ABA \\
\hline \multirow[t]{3}{*}{ Propionibacterium spp. } & & $13 \cdot 7$ & $7 / 7$ & WC, BA \\
\hline & P. acnes & $12 \cdot 4$ & $7 / 7$ & \\
\hline & P. granulosum & 1.3 & $2 / 7$ & \\
\hline Rothia sp. & R. mucilaginosa & 1.3 & $2 / 7$ & NA \\
\hline \multicolumn{5}{|l|}{ Firmicutes } \\
\hline \multirow{3}{*}{ Enterococcus spp. } & & 0.9 & $2 / 7$ & WC, NA, MC \\
\hline & E. faecalis & 0.4 & $1 / 7$ & \\
\hline & E. gallinarum & 0.4 & $1 / 7$ & \\
\hline \multirow{3}{*}{ Lactobacillus spp. } & & $2 \cdot 1$ & $2 / 7$ & WC, RCA \\
\hline & L. brevis & 1.7 & $1 / 7$ & \\
\hline & L. gasseri & 0.4 & $1 / 7$ & \\
\hline \multirow[t]{5}{*}{ Staphylococcus spp. } & & $60 \cdot 1$ & $7 / 7$ & WC, NA, MSA, MC, ABA \\
\hline & S. epidermidis & 51.9 & $7 / 7$ & \\
\hline & S. lugdunensis & $5 \cdot 6$ & $3 / 7$ & \\
\hline & S. aureus/haemolyticus/hominis & 1.7 & $3 / 7$ & \\
\hline & S. pasteuri/warneri & 0.9 & $2 / 7$ & \\
\hline \multirow[t]{3}{*}{ Streptococcus spp. } & & $17 \cdot 6$ & $7 / 7$ & $W C, N A, A B A, B A$ \\
\hline & S. salivarius/thermophilus/vestibularis & $11 \cdot 6$ & $7 / 7$ & \\
\hline & S. mitis/pneumoniae & 3.4 & $3 / 7$ & \\
\hline Veillonella sp.† & V. atypical/dispar/parvula† & 1.7 & $2 / 7$ & WC \\
\hline \multicolumn{5}{|l|}{ Proteobacteria } \\
\hline Escherichia/Shigella spp. & & 0.4 & $1 / 7$ & MSA \\
\hline Klebsiella pneumoniae & & 0.4 & $1 / 7$ & WC \\
\hline
\end{tabular}

$11 \%$, respectively), while, furthermore, the Acidobacteria phylum was detected at subdominant levels $(<1 \%)$ (Fig. 2(a)).

At the family level, the most abundant phylum, Firmicutes, was composed of Streptococcaceae and Staphylococcaceae (approximately 13 and $10 \%$, respectively), i.e. families whose members are facultative anaerobes. However, surprisingly, families comprising obligate anaerobic, gut-associated members were represented at relatively high relative abundances, i.e. Lachnospiraceae, Ruminococcaceae, as well as members of the Clostridiales Incertae Sedis XIV (Fig. 2(b)). The second most abundant phylum, Proteobacteria, comprised members of the four families, mainly Pseudomonadaceae and Burkholderiaceae (approximately 17 and 10\%, respectively), while Caulobacteraceae and Xanthomonadaceae were
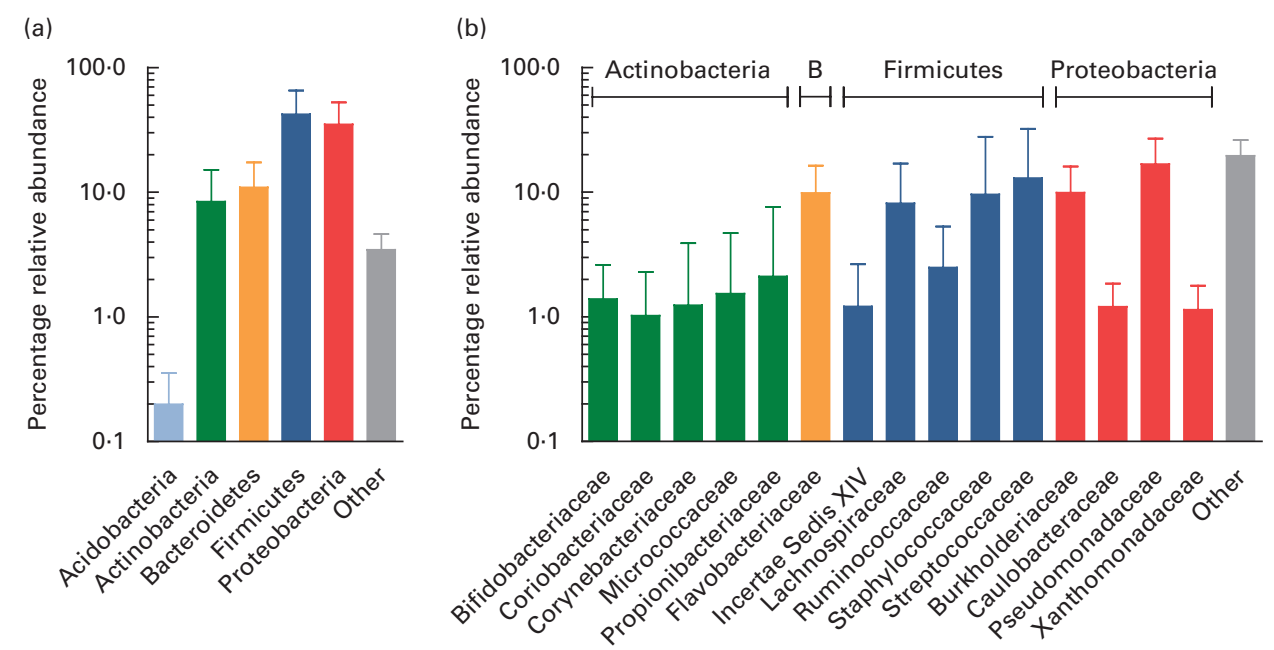

Fig. 2. Mean relative $16 \mathrm{~S}$ ribosomal RNA gene abundances detected in breast milk at (a) the phylum level and (b) the family level ( $>1 \%$ mean relative abundance), using pyrosequencing. Values are means for all seven mothers at all three sampling points, with standard deviations represented by vertical bars (n 21). B, Bacteroidetes. 


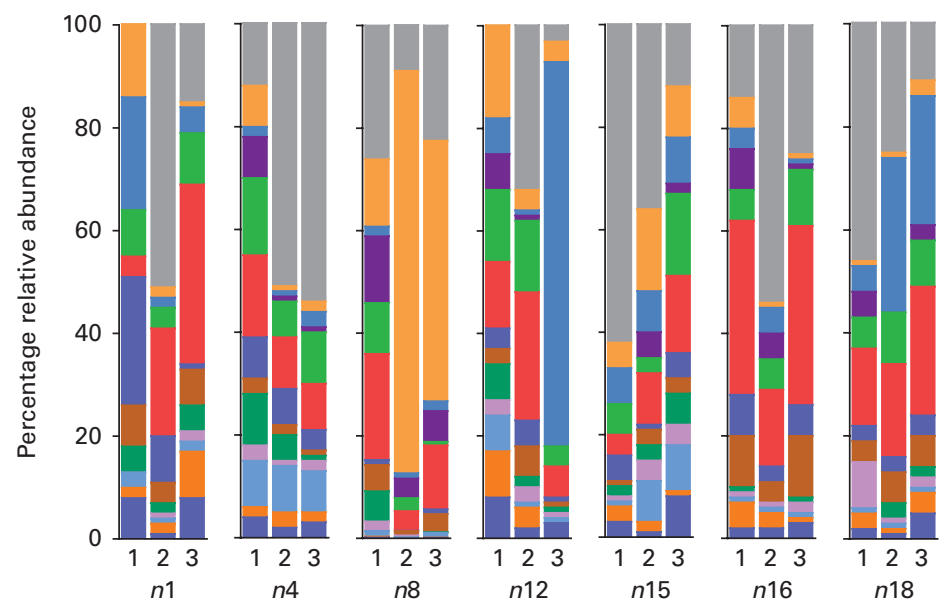

Fig. 3. Relative $16 \mathrm{~S}$ ribosomal RNA gene abundances of the twelve predominant genera detected in $\geq 90 \%$ of individual samples ( $n$ 21) at a mean relative abundance $>1 \%$, and observed in at least one of the three breast milk samples collected from each of the seven mothers (except six of the seven mothers for Rothia, fifteen of the twenty-one samples), using pyrosequencing. ${ }_{\square}$, Bifidobacterium; $\_$, Blautia; $\_$, Brevundimonas; $\square$, Burkholderia; $\square$, Coryne-

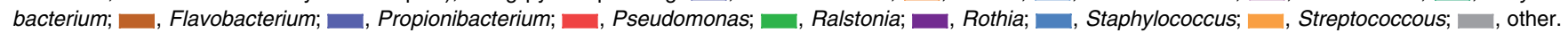
Indexes 1,2 and 3 , sampling points at days $3-6,9-14$ and $25-30$ postpartum, respectively.

detected at lower relative abundances $(<2 \%)$. Flavobacteriaceae was the only family detected at high relative abundance within the phylum Bacteroidetes (approximately 10\%). The phylum Actinobacteria was largely composed of Propionibacteriaceae, Bifidobacteriaceae, Coriobacteriaceae, Corynebacteriaceae and Micrococcaceae, each at relative abundances $\leq 2 \%$.

At the genus level, at total of 193 genera were detectable (i.e. $>0.01 \%$ relative abundance) in at least one sample. However, twelve genera appeared to be predominant in the breast milk ecosystem, as they were detected in $\geq 90 \%$ of individual samples with a mean relative abundance $>1 \%$, and observed in the samples of all seven mothers (except for Rothia, which was detected in six of the seven mothers and fifteen of the twenty-one samples). The relative abundances of these predominant genera were individual-specific and subject to intra-individual variations over time, such as the large fluctuations of Streptococcus and Staphylococcus over time observed in the profiles of subjects $n 8$ and $n 12$, respectively (Fig. 3).

Overall, the highest mean relative abundance was observed for the genus Pseudomonas (approximately 17\%), followed by the genera Streptococcus, Staphylococcus and Ralstonia, each at a mean relative abundance between 8 and 13\% (Fig. 4(a)). While the high relative abundances of Streptococcus and Staphylococcus confirm the results of the culture-dependent approach, no strains of Pseudomonas or Ralstonia (previously included in the genus Pseudomonas) were isolated by culture, although MC agar medium is expected to support their growth. Furthermore, the presence of Bifidobacterium, Propionibacterium as well as Rothia at lower relative abundances is well supported by culture and isolation of the respective genera (Table 1). In contrast, although few Lactobacillus strains were isolated by culture, this genus was detected at a mean relative abundance below $0 \cdot 1 \%$ only.

While, using culture, Veillonella spp. were identified as gutassociated obligate anaerobes belonging to the Clostridia class in the samples of two mothers, this genus was also detected at low abundance in four of the twenty-one samples from three mothers using pyrosequencing. Furthermore, the anaerobic genus Blautia (Clostridia class) was detected in nineteen of the twenty-one samples at a mean relative abundance of $1.2 \%$ (Fig. 4(a)). At the subdominant level ( $0 \cdot 11 \%$ mean relative abundance), sixteen genera were observed in at least one breast milk sample of each mother, and in sixteen or more of the twenty-one individual samples. The identified genera comprised typical gut-associated obligate anaerobes (6.4\% relative abundance), such as Bacteroides and multiple members of the Clostridia, including besides the genera Dorea and Ruminococcus, major butyrate producers, such as Faecalibacterium and Roseburia, as well as Coprococcus and Subdoligranulum (Fig. 4(b)).

\section{Discussion}

The aim of the present study was to investigate whether breast milk represents a source of bacterial diversity, including major gut-associated anaerobes such as members of Bacteroidetes and Clostridia, influencing the initial gut colonisation of the breast-fed neonate. Therefore, aerobic and anaerobic culture methods were successfully combined with state-of-the-art, culture-independent methods, including Sanger sequencing and high-throughput sequencing (i.e. 454-pyrosequencing).

Culture on non-selective agar media revealed that breast milk harboured low mean viable bacterial counts $<$ log $3 \mathrm{cfu} / \mathrm{ml}$, which is in agreement with previous reports using culture-dependent methods ${ }^{(4,8)}$. However, Solis et al. ${ }^{(5)}$ reported mean values of $\log 3-5 \mathrm{cfu} / \mathrm{ml}$ for samples from healthy mothers, which may be attributed to differences in culture conditions and/or to geographical factors. Furthermore, the authors have observed decreasing bacterial densities in breast milk over time, while this was not confirmed in the present study, where no decreasing or increasing trend in 

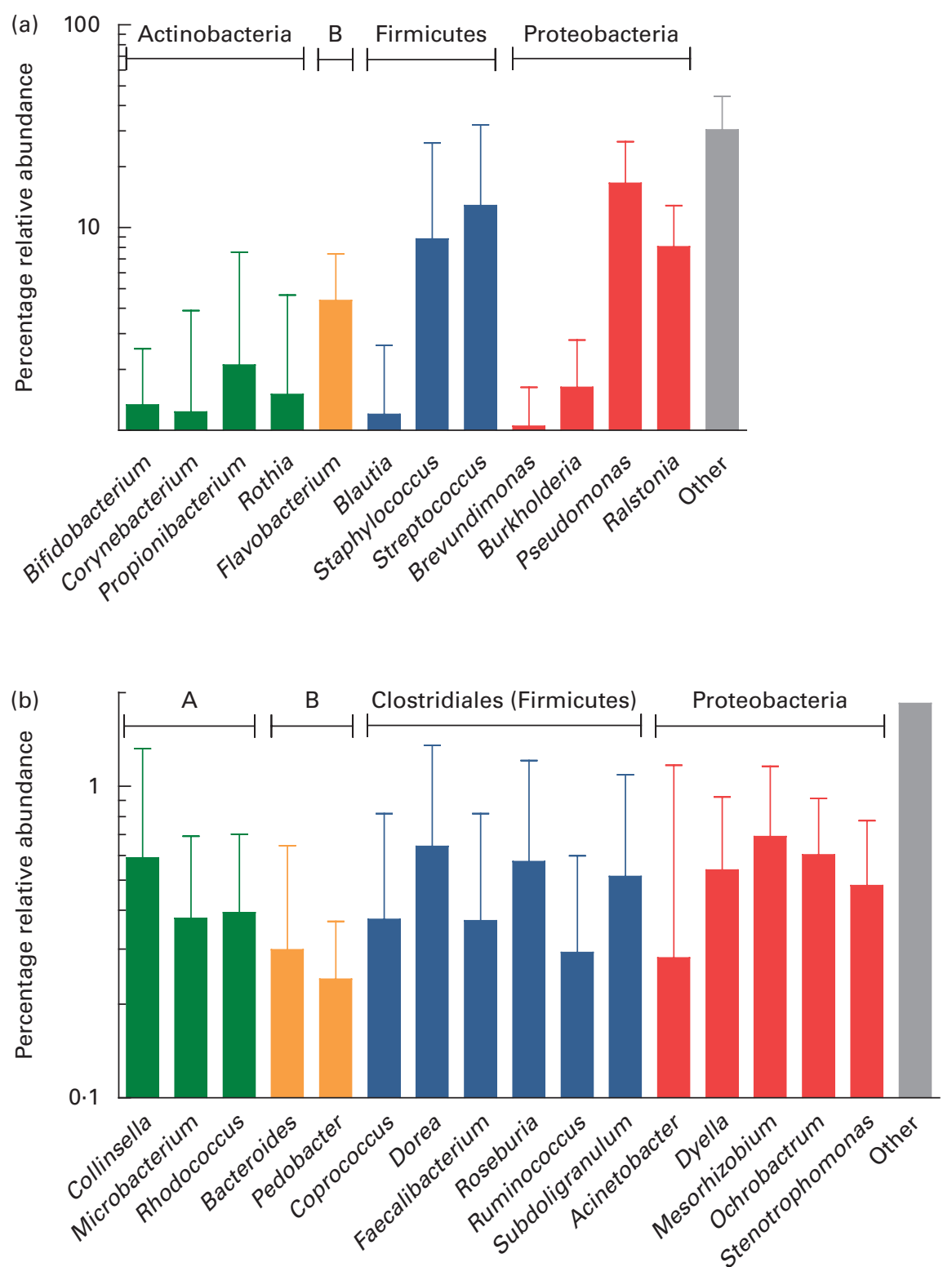

Fig. 4. (a) Mean relative $16 \mathrm{~S}$ ribosomal RNA gene abundances of the twelve predominant genera detected in $\geq 90 \%$ of individual samples at a mean relative abundance $>1 \%$, and observed in at least two of the three breast milk samples from each of the seven mothers (except six of the seven mothers for Rothia, fifteen of the twenty-one samples), using pyrosequencing. (b) Mean relative 16S ribosomal RNA gene abundances of the sixteen subdominant genera detected at a mean relative abundance between 0.1 and $1 \%$ and present in at least one breast milk sample from each mother, and in more than two of the three individual samples. Anaerobes: (a) Bifidobacterium and Blautia; (b) Collinsella, Bacteroides, Coprococcus, Dorea, Faecalibacterium, Roseburia, Ruminococcus and Subdoligranulum. Butyrate producers: Coprococcus, Faecalibacterium, Roseburia and Subdoligranulum. Values are means for all seven mothers at all three sampling points, with standard deviations represented by vertical bars (n 21). A, Actinobacteria; B, Bacteroidetes.

individual bacterial populations was observed within the first month postpartum.

Using partial 16S rRNA sequencing, the vast majority of isolated strains was assigned to the genus Staphylococcus, followed by the strains of Streptococcus and Propionibacterium, and thus showing that facultative anaerobic members of Firmicutes and Actinobacteria predominate in the breast milk ecosystem, while members of Proteobacteria and Bacteroidetes were few or not detected, respectively. Results obtained from the quantification of presumptive populations, however, should be taken with caution due to medium selectivity/electivity bias inherent to culture and the fact that non-fastidious facultative anaerobes are favoured over more fastidious obligate anaerobes, which may thus have escaped culture. For instance, Staphylococcus spp. were able to grow on most culture media, and it can be assumed that they accounted for a high percentage of presumptive total facultative anaerobes and total anaerobes, and consequently that the population of presumptive Streptococcus has been overestimated. However, despite the fact that facultative anaerobes are able to grow in strict anaerobic conditions, the presence of Bifidobacterium as well as Veillonella spp., isolated from 
the samples of two different mothers each, demonstrates that breast milk can be a source of viable obligate gut-associated anaerobes.

While to our knowledge, no Veillonella spp. have been isolated from breast milk in previous research, all other identified taxa, including the less frequently isolated genera, Rothia, Enterococcus, Escherichia/Shigella and Klebsiella, have been detected in previous studies, although with varying frequencies $^{(4,6)}$. The predominance of Staphylococcus, Streptococcus and Propionibacterium spp. largely confirms the findings of previous culture-dependent studies ${ }^{(4-6)}$. However, in other studies, Enterococcus spp. have also been identified among the dominant populations ${ }^{(5,6)}$, while this genus was only identified in two samples in the present study, despite the use of MC agar selective for Enterococcus spp. and Enterobacteriaceae. The presence of Enterococcus spp. in breast milk appears to be largely variable, which is corroborated by quantitative PCR analyses, quantifying this population at $0.5-4 \%$ of the total bacterial DNA ${ }^{(14,16)}$. In contrast to previous research, no viable strains belonging to genera such as Actinomyces and Corynebacterium (Actinobacteria phylum) ${ }^{(4,8)}$, Lactococcus and Leuconostoc (Bacilli class) ${ }^{(4)}$, as well as to the obligate anaerobic genus Peptostreptococcus (Clostridia class) ${ }^{(8)}$ were isolated by culture; however, these genera were identified as minor components of breast milk microbiota only in these studies.

While culture and 16S rRNA sequencing of the isolates allowed for characterising the major viable bacterial populations in breast milk, pyrosequencing demonstrated that bacterial diversity in breast milk is by far higher than measured by culture-dependent methods, as well as by culture-independent methods with much lower throughput similar to the generation of $16 \mathrm{~S}$ rRNA clone libraries ${ }^{(15)}$. Taxonomic assignment of the sequences using the Ribosomal Database Project Classifier revealed that, on average, the highest relative abundance was observed for the Firmicutes phylum and, more specifically, the genera Staphylococcus and Streptococcus, which largely supports the findings of our culture-dependent approach. However, in contrast to culture, using pyrosequencing, the relative abundance of Streptococcus was slightly higher than that for Staphylococcus, which may be explained by more fastidious growth requirements of Streptococcus strains.

The second most abundant phylum, Proteobacteria, was largely made up of the genera Pseudomonas and Ralstonia, which is supported by previous culture-independent studies $^{(15,17)}$. However, surprisingly, no corresponding strains were detected in our culture-dependent approach, although both MC and nutrient agar are expected to support the growth of such non-fastidious organisms. This suggests that the detected DNA could correspond to stressed, dead or (partially) lysed cells, possibly through the action of antimicrobial components, leucocytes and/or weakness for competition against other bacteria of the breast milk ecosystem. For instance, Lactobacillus spp. isolated from breast milk have been reported as capable of inhibiting nosocomial pathogens, such as strains of Pseudomonas, Escherichia and Serratia ${ }^{(29)}$.
In concordance with culture, the Actinobacteria phylum comprised mainly the genus Propionibacterium, and a lower relative abundance of Rothia and Bifidobacterium populations. Hunt et al. ${ }^{(17)}$ observed a similar bacterial diversity in terms of the fifteen predominant genera in breast milk when using pyrosequencing, but detected only a few sequences of Bifidobacterium, while in the present study, Bifidobacterium was among the twelve most abundant genera. Also, Cabrera-Rubio et al. ${ }^{(16)}$ did not report any pyrosequences belonging assigned to Bifidobacterium. However, the presence of DNA from Bifidobacterium in the present study is supported by previous quantitative PCR analyses quantifying this population in the range of log 2-6 genome equivalents $/ \mathrm{ml}$, corresponding to up to $66 \%$ of the total bacterial genome equivalents ${ }^{(14,16,30)}$. Besides geographical, dietary, host and sampling time factors, different outcomes in pyrosequencing analyses may be due to differences in methodological factors, such as DNA extraction procedures $^{(31,32)}$, PCR bias and different taxonomic classification accuracies due to the use of primers targeted at different tandem variable $16 \mathrm{~S}$ rRNA gene regions ${ }^{(33,34)}$ (i.e. the V1-V2 region in both previous studies ${ }^{(16,17)}$, compared with the V5-V6 region in the present study), as well as a higher depth of sequencing in the present study (i.e. average 5051 reads/sample) compared with both previous studies (i.e. average $2623^{(16)}$ and $\left.3400 \mathrm{reads} / \mathrm{sample}^{(17)}\right)$. This may also explain further differences, such as the higher relative abundance of Corynebacterium and Serratia in some of the samples analysed by Hunt et $a l^{(17)}$, as well as the predominance of Weissella and Leuconostoc over Staphylococcus and Streptococcus in the study by Cabrera-Rubio et al. ${ }^{(16)}$. Furthermore, a higher relative abundance of the anaerobic genera Prevotella and Veillonella was observed in both previous pyrosequencing studies ${ }^{(16,17)}$; however, this may be due to the fact that samples were collected at a later stage (i.e. 1-10 months postpartum), when compared with within the first month postpartum in the present study.

Much focus has been given to the presence of the potential probiotic Lactobacillus spp. in breast $\operatorname{milk}^{(5,7,29,35,36)}$. However, the mean relative abundance of Lactobacillus was generally low in the present study group (i.e. $<0 \cdot 1 \%$ ), which is in accordance with the low isolation frequency of Lactobacillus spp. (i.e. L. gasseri and L. brevis) observed using culture. Therefore, the supplementation of formulae with probiotic strains of Lactobacillus may not reflect the natural bacterial inoculum transferred to the breast-fed neonate, despite their potential for disease prevention and treatment ${ }^{(37)}$. Differences in bacterial densities may, however, be attributed to geographical factors, including dietary habits, such as the consumption of probiotic products. In this regard, it has been shown that Lactobacillus spp. when administered orally can be recovered from breast milk ${ }^{(38-40)}$ and thus influence its composition.

Pyrosequencing largely confirmed the culture-dependent approach by the detection of Staphylococcus, Streptococcus and Propionibacterium spp. as dominant populations, which as facultative anaerobic skin/oral-associated populations may be transferred to the breast by secondary contamination 
such as via suckling. Interestingly, however, pyrosequencing also allowed for detecting a series of obligate anaerobic, gut-associated genera, such as Bacteroides and multiple members of the Clostridia class, in addition to Bifidobacterium and Veillonella also identified by culture. Among the members of the Clostridia, genera such as Blautia, Dorea and Ruminococcus were detected for the first time and, moreover, surprisingly, also major butyrate producers important for colonic health, such as Faecalibacterium, Roseburia, Coprococcus and Subdoligranulum. The present findings are supported by and extend previous quantitative PCR analyses confirming, although at a higher taxonomic level only, the presence of the Bacteroides group, the Clostridium coccoides group, as well as the Clostridium clusters IV and XIV that comprise the major gut-associated butyrate producers, Faecalibacterium and Roseburia, respectively ${ }^{(14,16)}$. Thus, breast milk may represent a source of such obligate anaerobes to the breast-fed neonate and may contribute to the colonisation process, as suggested in our previous research ${ }^{(13)}$ by the presence of Bifidobacterium, Bacteroides and Veillonella spp. as pioneer bacteria in corresponding neonatal faeces. We hypothesise that Veillonella spp., as non-carbohydrate fermenting, lactate utilisers (in concert with Propionibacterium spp.), may play an important function in the breast milk ecosystem by metabolising lactate resulting from lactose fermentation by predominant breast milk populations, to propionate and acetate ${ }^{(41)}$. These trophic functions may be transferred to the breast-fed neonate and contribute to the colonisation and gut maturation process. Furthermore, the detection of the members of Bacteroidetes and Clostridia in breast milk suggests that they originate from the maternal gut, since such obligate anaerobes do not proliferate outside their host. This hypothesis, i.e. the translocation of maternal bacteria to subsequently reach the mammary gland via a bacterial enteromammary pathway, has been proposed previously ${ }^{(8,42)}$ and will be the object of future research.

Furthermore, it remains to be elucidated whether the series of anaerobes detected using pyrosequencing escaped culture due to their low abundance in breast milk and/or due to fastidious growth requirements (viable but not culturable with the applied methods), and/or the presence of antimicrobial compounds, or if dead cells or parts thereof are transferred to the breast-fed neonate, which nevertheless could elicit an immune response and affect neonatal development. Although, in the present study, samples were processed under anaerobic conditions using culture media supporting the growth of gutassociated anaerobes, a higher number of isolates may be necessary for identifying subdominant populations. On the other hand, highly fastidious anaerobes may be recovered using more laborious roll-tube techniques developed by Hungate $^{(43)}$ and approaches like the recently described 'microbial culturomics $^{(44)}$ that rely on a vast array of culture conditions.

\section{Acknowledgements}

The present study was supported by Nestlé Nutrition (Vevey, Switzerland), Nestec (Lausanne, Switzerland) and the Swiss Foundation for Nutrition Research (SFEFS) (Zurich,
Switzerland) for the purpose of basic research only and is not related to any patent, product in development or marketed product. None of the authors are employees or consultants of the funders. The funders had no role in study design, data collection and analysis, decision to publish, or preparation of the manuscript.

The authors are grateful to Patrick Bühr, Michael Friedt, Petra Martel, Daniela Rogler and Rebekka Koller at the University Children's Hospital (Zurich, Switzerland) for their effort in volunteer recruitment and sampling, to Peter Frei and Tania Torossi for their assistance in 16S rRNA gene sequencing, carried out at the Genetic Diversity Centre of ETH Zurich (Zurich, Switzerland), as well as to Medela AG (Baar, Switzerland) for providing electrical breast pumps throughout the study.

C. B., C. L., C. C. and T. J. conceived and designed the experiments. T. J. and C. C. acquired the data. T. J., C. C., C. L. and C. B. analysed and interpreted the data. T. J., C. C., C. L. and C. B. wrote and approved the manuscript. The authors declare that no competing interests exist.

\section{References}

1. Hosea Blewett HJ, Cicalo MC, Holland CD, et al. (2008) The immunological components of human milk. In Advances in Food and Nutrition Research, vol. 54, pp. 45-80 [LT Steve, editor]. San Diego: Academic Press.

2. Fernandez L, Langa S, Martin V, et al. (2013) The human milk microbiota: origin and potential roles in health and disease. Pharmacol Res 69, 1-10.

3. Rautava S, Luoto R, Salminen S, et al. (2012) Microbial contact during pregnancy, intestinal colonization and human disease. Nat Rev Gastroenterol Hepatol 9, 565-576.

4. Heikkila MP \& Saris PE (2003) Inhibition of Staphylococcus aureus by the commensal bacteria of human milk. $J$ Appl Microbiol 95, 471-478.

5. Solis G, de Los Reyes-Gavilan CG, Fernandez N, et al. (2010) Establishment and development of lactic acid bacteria and bifidobacteria microbiota in breast-milk and the infant gut. Anaerobe 16, 307-310.

6. Jimenez E, Delgado S, Maldonado A, et al. (2008) Staphylococcus epidermidis: a differential trait of the fecal microbiota of breast-fed infants. BMC Microbiol 8, 143.

7. Albesharat R, Ehrmann MA, Korakli M, et al. (2011) Phenotypic and genotypic analyses of lactic acid bacteria in local fermented food, breast milk and faeces of mothers and their babies. Syst Appl Microbiol 34, 148-155.

8. Perez PF, Dore J, Leclerc M, et al. (2007) Bacterial imprinting of the neonatal immune system: lessons from maternal cells? Pediatrics 119, e724-e732.

9. Arboleya S, Ruas-Madiedo P, Margolles A, et al. (2010) Characterization and in vitro properties of potentially probiotic Bifidobacterium strains isolated from breast-milk. Int J Food Microbiol 149, 28-36.

10. Martin V, Maldonado-Barragan A, Moles L, et al. (2012) Sharing of bacterial strains between breast milk and infant feces. J Hum Lact 28, 36-44.

11. Martin R, Jimenez E, Heilig H, et al. (2009) Isolation of bifidobacteria from breast milk and assessment of the bifidobacterial population by PCR-denaturing gradient gel electrophoresis and quantitative real-time PCR. Appl Environ Microbiol 75 , 965-969 
12. Makino H, Kushiro A, Ishikawa E, et al. (2011) Transmission of intestinal Bifidobacterium longum subsp. longum strains from mother to infant, determined by multilocus sequencing typing and amplified fragment length polymorphism. Appl Environ Microbiol 77, 6788-6793.

13. Jost T, Lacroix C, Braegger CP, et al. (2012) New insights in gut microbiota establishment in healthy breast fed neonates. PLoS One 7, e44595.

14. Collado MC, Delgado S, Maldonado A, et al. (2009) Assessment of the bacterial diversity of breast milk of healthy women by quantitative real-time PCR. Lett Appl Microbiol 48, 523-528.

15. Martin R, Heilig HGHJ, Zoetendal EG, et al. (2007) Cultivation-independent assessment of the bacterial diversity of breast milk among healthy women. Res Microbiol 158, 31-37.

16. Cabrera-Rubio R, Collado MC, Laitinen K, et al. (2012) The human milk microbiome changes over lactation and is shaped by maternal weight and mode of delivery. $A m \mathrm{~J}$ Clin Nutr 96, 544-551.

17. Hunt KM, Foster JA, Forney LJ, et al. (2011) Characterization of the diversity and temporal stability of bacterial communities in human milk. PLoS One 6, e21313.

18. Macfarlane GT, Hay S, Macfarlane S, et al. (1990) Effect of different carbohydrates on growth, polysaccharidase and glycosidase production by Bacteroides ovatus, in batch and continuous culture. J Appl Bacteriol 68, 179-187.

19. Beerens H (1991) Detection of bifidobacteria by using propionic acid as a selective agent. Appl Environ Microbiol $\mathbf{5 7}, 2418-2419$

20. Steer TE, Gee JN, Johnson IT, et al. (2004) Biodiversity of human faecal bacteria isolated from phytic acid enriched chemostat fermenters. Curr Issues Intest Microbiol 5, 23-39.

21. Hartemink R, Domenech VR \& Rombouts FM (1997) LAMVAB - a new selective medium for the isolation of lactobacilli from faeces. J Microbiol Methods 29, 77-84.

22. Dethlefsen L, Huse S, Sogin ML, et al. (2008) The pervasive effects of an antibiotic on the human gut microbiota, as revealed by deep $16 \mathrm{~S}$ rRNA sequencing. PLoS Biol 6, e280.

23. Altschul SF, Gish W, Miller W, et al. (1990) Basic local alignment search tool. J Mol Biol 215, 403-410.

24. Benson DA, Karsch-Mizrachi I, Lipman DJ, et al. (2011) GenBank. Nucleic Acids Res 39, D32-D37.

25. Andersson AF, Lindberg M, Jakobsson H, et al. (2008) Comparative analysis of human gut microbiota by barcoded pyrosequencing. PLoS One 3, e2836.

26. Wang Q, Garrity GM, Tiedje JM, et al. (2007) Naive Bayesian classifier for rapid assignment of rRNA sequences into the new bacterial taxonomy. Appl Environ Microbiol 73, 5261-5267.

27. Schloss PD, Westcott SL, Ryabin T, et al. (2009) Introducing mothur: open-source, platform-independent, communitysupported software for describing and comparing microbial communities. Appl Environ Microbiol 75, 7537-7541.

28. Chao A \& Shen T-J (2003) Nonparametric estimation of Shannon's index of diversity when there are unseen species in sample. Environ Ecol Stat 10, 429-443.

29. Jara S, Sanchez M, Vera R, et al. (2011) The inhibitory activity of Lactobacillus spp. isolated from breast milk on gastrointestinal pathogenic bacteria of nosocomial origin. Anaerobe 17, 474-477.

30. Gronlund MM, Gueimonde M, Laitinen K, et al. (2007) Maternal breast-milk and intestinal bifidobacteria guide the compositional development of the Bifidobacterium microbiota in infants at risk of allergic disease. Clin Exp Allergy 37, 1764-1772

31. Yuan S, Cohen DB, Ravel J, et al. (2012) Evaluation of methods for the extraction and purification of DNA from the human microbiome. PLoS One 7, e33865.

32. Maukonen J, Simoes C \& Saarela M (2012) The currently used commercial DNA-extraction methods give different results of clostridial and actinobacterial populations derived from human fecal samples. FEMS Microbiol Ecol 79, 697-708.

33. Claesson MJ, Wang Q, O'Sullivan O, et al. (2010) Comparison of two next-generation sequencing technologies for resolving highly complex microbiota composition using tandem variable $16 \mathrm{~S}$ rRNA gene regions. Nucleic Acids Res 38, e200.

34. Wu GD, Lewis JD, Hoffmann C, et al. (2010) Sampling and pyrosequencing methods for characterizing bacterial communities in the human gut using $16 \mathrm{~S}$ sequence tags. BMC Microbiol 10, 206.

35. Martin R, Langa S, Reviriego C, et al. (2003) Human milk is a source of lactic acid bacteria for the infant gut. J Pediatr 143, $754-758$.

36. Martin R, Jimenez E, Olivares M, et al. (2006) Lactobacillus salivarius CECT 5713, a potential probiotic strain isolated from infant feces and breast milk of a mother-child pair. Int J Food Microbiol 112, 35-43.

37. Thomas DW \& Greer FR (2010) Probiotics and prebiotics in pediatrics. Pediatrics 126, 1217-1231.

38. Jimenez E, Fernandez L, Maldonado A, et al. (2008) Oral administration of Lactobacillus strains isolated from breast milk as an alternative for the treatment of infectious mastitis during lactation. Appl Environ Microbiol 74, 4650-4655.

39. Arroyo R, Martin V, Maldonado A, et al. (2010) Treatment of infectious mastitis during lactation: antibiotics versus oral administration of Lactobacilli isolated from breast milk. Clin Infect Dis 50, 1551-1558.

40. Abrahamsson TR, Sinkiewicz G, Jakobsson T, et al. (2009) Probiotic lactobacilli in breast milk and infant stool in relation to oral intake during the first year of life. $J$ Pediatr Gastroenterol Nutr 49, 349-354

41. Duncan SH, Louis P \& Flint HJ (2004) Lactate-utilizing bacteria, isolated from human feces, that produce butyrate as a major fermentation product. Appl Environ Microbiol 70, $5810-5817$.

42. Martín R, Langa S, Reviriego C, et al. (2004) The commensal microflora of human milk: new perspectives for food bacteriotherapy and probiotics. Trends Food Sci Technol 15, $121-127$.

43. Hungate RE (1969) A roll tube method for cultivation of strict anaerobes. In Methods in Microbiology, vol. 3, pp. 117-132 [JR Norris and DW Ribbons, editors]. New York: Academic Press.

44. Lagier JC, Armougom F, Million M, et al. (2012) Microbial culturomics: paradigm shift in the human gut microbiome study. Clin Microbiol Infect 18, 1185-1193. 